\title{
Smartphone use is a risk factor for pediatric dry eye disease according to region and age: a case control study
}

Jun Hyung Moon, Kyoung Woo Kim and Nam Ju Moon ${ }^{*}$

\begin{abstract}
Background: In 2014, the overall rate of smartphone use in Korea was 83 and $89.8 \%$ in children and adolescents. The rate of smartphone use differs according to region (urban vs. rural) and age (younger grade vs. older grade). We investigated risk and protective factors associated with pediatric dry eye disease (DED) in relation to smartphone use rate according to region and age.

Methods: We enrolled 916 children and performed an ocular exam that included slit lamp exam and tear break-up time. A questionnaire administered to children and their families consisted of video display terminal (VDT) use, outdoor activity, learning, and modified ocular surface disease index (OSDI) score. DED was defined based on the International Dry Eye Workshop guidelines (Objective signs: punctate epithelial erosion or short tear break-up time; subjective symptoms: modified OSDI score) We performed statistical analysis of risk factors and protective factors in children divided into groups as follows: DED vs. control, urban vs. rural, younger grade (1st to 3rd) vs. older grade (4th to 6th).

Results: A total of $6.6 \%$ of children were included in the DED group, and $8.3 \%$ of children in the urban group were diagnosed with DED compared to $2.8 \%$ in the rural group $(P=0.03)$. The rate of smartphone use was $61.3 \%$ in the urban group and $51.0 \%$ in the rural group $(P=0.04)$. In total, $9.1 \%$ of children in the older-grade group were diagnosed with DED compared to $4 \%$ in the younger-grade group ( $P=0.03)$. The rate of smartphone use was $65.1 \%$ in older-grade children and $50.9 \%$ in younger-grade children $(P<0.001)$. The mean daily duration of smartphone use was longer in the DED group than controls (logistic regression analysis, $P<0.001, O R=13.07$ ), and the mean daily duration of outdoor activities was shorter in the DED group than controls (logistic regression analysis, $P<0$. $01, \mathrm{OR}=0.33$ ). After cessation of smartphone use for 4 weeks in the DED group, both subjective symptoms and objective signs had improved.
\end{abstract}

Conclusions: Smartphone use in children was strongly associated with pediatric DED; however, outdoor activity appeared to be protective against pediatric DED. Older-grade students in urban environments had DED risk factors (long duration of smartphone use), and a short duration of outdoor activity time. Therefore, close observation and caution are needed when older children in urban areas use smartphones.

Keywords: Dry eye disease, Pediatrics, Smartphone, Outdoor activity, Video display terminal

\footnotetext{
* Correspondence: njmoon@chol.com

Department of Ophthalmology, College of Medicine, Chung Ang University

Hospital, 102, Heukseok-ro, Dongjak-gu, Seoul 06973, Korea
} 


\section{Background}

Dry eye disease (DED) can occur in association with a number of congenital, autoimmune, endocrine, and inflammatory disorders, or under certain environmental and nutritional conditions [1]. We initiated this study to investigate environmental risk factors and protective factors for pediatric dry eye disease to correct risk factors and encourage protective factors.

Korea has one of the world's highest smartphone use rates with an estimated $89.8 \%$ rate of usage in 2014 . Pediatric dry eye disease tends to have a higher prevalence in younger ages [1]. Therefore, it is imperative to investigate risk factors and protective factors for pediatric DED to prevent disease progression as much as possible.

Use of video display terminals and learning (reading and writing) for long hours has been associated with a decreased maximum blink interval, hence the development of dry eye symptoms [2-4]. In addition, many people report ocular fatigue after prolonged work with video display terminals, in part prompting legislation regulating the use of these terminals [5].

Pediatric DED is a public health concern and economic challenge; thus, it is important to dedicate considerable clinical attention to pediatric DED in Korea [6]. In our previous study, we investigated the association between pediatric DED and video display terminal use [7]. DED is a multi-factorial disease, and there have not yet been any in-depth studies considering risk factors and protective factors for pediatric DED according to age and region. In our study, we investigated risk factors and protective factors for pediatric dry eye disease associated with smartphone use rate according to region and age.

\section{Methods}

\section{Subjects}

This study was a school-based cross sectional, casecontrol study. We performed ophthalmologic examinations on 630 children from eight primary schools in Seoul, Korea (urban, male:female $=318: 312$ ) and in 286 children in Paju, Korea (rural, male:female $=168: 118$ ) from May through October 2015. Seoul is the capital city of South Korea and a large urban center with a population of approximately 10 million. Paju is a city with a population near 400,000. We performed ocular exams on children in younger (1st to 3rd grade, aged 7 to 9) and older (4th to 6th grade, aged 10 to 12 ) grades from elementary schools in these two regions.

This study was performed according to the Declaration of Helsinki on Biomedical Research Involving Human Subjects. The Institutional Review Board of Chung Ang University of Medicine Review Board approved the clinical study. Both parents and children themselves provided written informed consent after being given a detailed explanation of the study. We confirmed that parents had seen the manuscript and patient data and agreed to its publication in a journal.

The relative humidity in this region of Korea during the time of the study (May to October) ranged from 57.8 to $58.2 \%$ in urban areas (Seoul) and 58.1 to $58.4 \%$ in rural areas (Paju). There was no significant difference in relative humidity between the two regions.

Exclusion criteria were: (1) children who underwent any type of eye surgery in the past 6 months, (2) children who had nocturnal lagophthalmos, (3) children who had eyelid problems (trichiasis, districhiasis, or epiblepharon), (4) children who had allergic conjunctivitis with the use of antihistamine drugs, (5) children who wear contact lenses, and (6) children who had congenital, endocrinal, or autoimmune disease.

\section{Ocular examinations}

A single examiner performed all ocular exams. These included visual acuity tests (best corrected VA with trial lens, LogMAR), autokeratomery, slit-lamp examinations of the cornea and conjunctiva, and evaluation of eyelid problems, allergic conjunctivitis, and exposure keratitis. Autokeratometry was measured using Topcon Auto Kerato-Refractometer KR-8800 by a single examiner; we reported the mean value of the three repeated measurements.

Tear break-up time (TBUT) was measured with a fluorescein strip (Haag-Streit Inter- national, Koniz, Switzerland) coated with one drop of balanced salt solution (BSS; Alcon Laboratories, Inc., Fort Worth, TX). After applying the strip to the inferior conjunctival fornix, the participant resumed normal blinking for several seconds. After the fluorescein solution spread across the corneal surface, the participant was asked to keep his or her eye open until the first defect of the tear film occurred. TBUT was defined as the interval between the last complete blink and the first appearance of a dry spot on the pre-corneal surface of the tear film [8]. The procedure was repeated three times for each eye tested, with results reported as the mean value of the three measurements.

A single examiner evaluated punctate epithelial erosions (PEE) in the cornea and conjunctiva using a slit lamp according to the Oxford Scheme Panel from 0 to 5 grades [9]. The study protocol is included (Additional file 1).

\section{Questionnaires}

A self-administered questionnaire was given to the children and their parents and both children and their parents completed each questionnaire. Questionnaires were designed to obtain information regarding risk factors for dry eye disease, including the mean daily duration of video display terminal (smartphone, computer, television) use, learning (reading and writing), outdoor activities, and past 
history of allergic disease and antihistamine drug use. Subjective ocular symptoms were measured with the modified ocular surface disease index (OSDI) score. This index was an objective parameter for DED diagnosis. Modified OSDI scores range from 0 to 100 points, and higher scores indicate greater discomfort due to dry eye disease. We added the OSDI index and ophthalmologic questionnaire (Additional file 1).

\section{Diagnostic criteria of dry eye disease}

Dry eye disease was diagnosed using a combination of questionnaire data and clinical ophthalmologic testing that elicited information on signs and symptoms based on the 2007 Dry Eye Workshop (DEWS) guidelines [10].

Objective signs included (1) TBUT less than $10 \mathrm{~s}$ or (2) positive corneal and conjunctival fluorescein staining. A modified OSDI score greater than 20 points was used for a positive measure of subjective symptoms. Children who had one or more objective signs and more than 20 points on the modified OSDI score were analyzed in the DED group.

\section{Statistical analysis}

We divided the school children into two groups and made the following comparisons: DED vs. control, urban vs. rural, younger graders vs. older graders. We used multivariate logistic regression analysis to assess DED risk and protective factors. Then, during 4 weeks of smartphone cessation in the DED group, we compared before and after parameters. Statistical analyses were performed using SPSS for Windows (version 21; SPSS, Inc., Chicago, IL). Continuous variables were compared between groups using the Student's t-test. The chi-square test was used to compare non-continuous variables between the two groups. $P$ values less than 0.05 were considered statistically significant.

\section{Results}

\section{Comparative analysis between control and dry eye disease group}

The rate of DED in all children was $6.6 \%$. There were significant differences based on region of residence, mean age, visual acuity, and rate of eyeglass use between the two groups. There was no statistically significant difference in terms of sex or spherical equivalent (diopter) between the two groups (Table 1).

The rate of smartphone use was $55.4 \%$ in the control group and, $96.7 \%$ in the DED group $(P<0.001)$. Smartphone use time per day was $0.62 \pm 0.68 \mathrm{~h}$ in the control group and $3.18 \pm 0.97 \mathrm{~h}$ in the DED group $(P<0.001)$; computer use time per day was $0.76 \pm 0.34 \mathrm{~h}$ in the control group and $1.10 \pm 0.53 \mathrm{~h}$ in the DED group $(P<0.001)$. Learning time per day was $2.31 \pm 1.02 \mathrm{~h}$ in the control group and $3.10 \pm 0.50 \mathrm{~h}$ in the DED group $(P<0.001)$.
Table 1 Comparative analysis between the control and Dry Eye Disease (DED) Groups

\begin{tabular}{llll}
\hline & Control & DED & P \\
\hline Subjects (N, (\%)) & $856(93.4 \%)$ & $60(6.6 \%)$ & \\
Region of Urban (\%) & 67.5 & 86.7 & $0.03^{\mathrm{a}}$ \\
Age (years) & $9.49 \pm 1.00$ & $9.90 \pm 0.93$ & $0.03^{\mathrm{b}}$ \\
Sex of Female (\%) & 46.5 & 53.3 & $0.47^{\mathrm{a}}$ \\
Visual Acuity (LogMAR) & $0.33 \pm 0.46$ & $0.61 \pm 0.37$ & $<0.001^{\mathrm{b}}$ \\
SE (diopter) & $-2.05 \pm 2.15$ & $-2.55 \pm 1.84$ & $0.22^{\mathrm{b}}$ \\
Use of Glass (\%) & 38.1 & 63.3 & $0.01^{\mathrm{a}}$ \\
Related with DED Risk Factors & & & \\
Use of Smartphones (\%) & 55.4 & 96.7 & $<0.001^{\mathrm{a}}$ \\
Smartphone use/day (hours) & $0.62 \pm 0.68$ & $3.18 \pm 0.97$ & $<0.001^{\mathrm{b}}$ \\
Computer use/day (hours) & $0.76 \pm 0.34$ & $1.10 \pm 0.53$ & $<0.001^{\mathrm{b}}$ \\
TV use/day (hours) & $1.07 \pm 0.54$ & $1.18 \pm 0.40$ & $0.27^{\mathrm{b}}$ \\
Learning/day (hours) & $2.31 \pm 1.02$ & $3.10 \pm 0.50$ & $<0.001^{\mathrm{b}}$ \\
Related with DED Protective Factors & & $1.47 \pm 0.32$ & $<0.001^{\mathrm{b}}$ \\
Sleep/day (hours) & $7.88 \pm 0.86$ & $7.70 \pm 0.61$ & $0.14^{\mathrm{b}}$ \\
Outdoor activity/day (hours) & $2.27 \pm 1.12$ & & \\
\hline DED dry eye disease, SE spherical & & & \\
\hline
\end{tabular}

$D E D$ dry eye disease, $S E$ spherical equivalent, logMAR logarithm of the minimum angle of resolution, $T V$ television

a'By Pearson's Chi-square test, ${ }^{b}$ By independent $t$ test

Outdoor activity time per day was $2.27 \pm 1.12 \mathrm{~h}$ in the control group and $1.47 \pm 0.32 \mathrm{~h}$ in the DED group $(P<0.001)$.

Comparative analysis between urban and rural groups for pediatric DED

We performed a comparative analysis of DED in 630 school children in urban environments and 286 children in rural environments. Visual acuity (LogMAR) was worse in the urban group, and the rate of eyeglass use was higher in the urban group compared to the rural group (Table 2).

The prevalence of DED was $8.3 \%$ in the urban group and $2.8 \%$ in the rural group. This difference was statistically significant $(P=0.03)$. The rate of smartphone use between the two regions also differed significantly, with $61.3 \%$ in urban areas and $51.0 \%$ in rural areas $(P=0.04)$. The mean daily duration of smartphone use was $0.93 \pm 1.01 \mathrm{~h}$ in the urban group and $0.47 \pm 0.69 \mathrm{~h}$ in the rural group $(P<0.001)$, while that of computer use was $0.84 \pm 0.29 \mathrm{~h}$ in the urban group and $0.68 \pm 0.46 \mathrm{~h}$ in the rural group $(P<0.001)$. The mean daily duration of outdoor activity was $2.06 \pm 1.13 \mathrm{~h}$ in the urban group and $2.57 \pm 0.95 \mathrm{~h}$ in the rural group $(P<0.001)$. The average daily duration of learning was $2.50 \pm 0.95 \mathrm{~h}$ in the urban group and $2.05 \pm 1.07 \mathrm{~h}$ in the rural group $(P<0.001)$. Age, spherical equivalent, TV watching, and sleeping time did not differ significantly between the two groups (Table 2). 
Table 2 Comparative analysis of pediatric DED between urban and rural groups

\begin{tabular}{llll}
\hline & Urban & Rural & $P$ \\
\hline Subjects (N) & 630 & 286 & \\
Age (years) & $9.51 \pm 1.00$ & $9.52 \pm 1.00$ & $0.91^{\mathrm{b}}$ \\
Dry eye disease (\%) & 8.3 & 2.8 & $0.03^{\mathrm{a}}$ \\
Visual acuity (logMAR) & $0.42 \pm 0.57$ & $0.23 \pm 0.45$ & $<0.001^{\mathrm{b}}$ \\
SE (diopter) & $-2.13 \pm 2.34$ & $-1.98 \pm 1.58$ & $0.41^{\mathrm{b}}$ \\
Use of glass (\%) & 42.9 & 32.9 & $0.04^{\mathrm{a}}$ \\
Related with DED Risk Factors & & & \\
$\quad$ Use of smartphones (\%) & 61.3 & 51 & $0.04^{\mathrm{a}}$ \\
$\quad$ Smartphone use/day (hours) & $0.93 \pm 1.01$ & $0.47 \pm 0.69$ & $<0.001^{\mathrm{b}}$ \\
Computer use/day (hours) & $0.84 \pm 0.29$ & $0.68 \pm 0.46$ & $<0.001^{\mathrm{b}}$ \\
TV use/day (hours) & $1.05 \pm 0.48$ & $1.15 \pm 0.64$ & $0.08^{\mathrm{b}}$ \\
$\quad$ Learning/day (hours) & $2.50 \pm 0.95$ & $2.05 \pm 1.07$ & $<0.001^{\mathrm{b}}$ \\
Related with DED Protective Factors & & \\
$\quad$ Sleep/day (hours) & $7.88 \pm 0.84$ & $7.84 \pm 0.87$ & $0.57^{\mathrm{b}}$ \\
Outdoor activity/day (hours) & $2.06 \pm 1.13$ & $2.57 \pm 0.95$ & $<0.001^{\mathrm{b}}$
\end{tabular}

$D E D$ dry eye disease, SE spherical equivalent, logMAR logarithm of the minimum angle of resolution, $T V$ television

aBy Pearson's Chi-square test, ${ }^{\mathrm{b}}$ By independent $\mathrm{t}$ test

\section{Comparative analysis of pediatric DED between younger} (1st to $3 \mathrm{rd}$ ) and older (4th to 6 th) grade children

We performed comparative analysis of DED in 452 younger grade (1st to $3 \mathrm{rd}$ ) children and 464 older grade (4th to 6th) children. The visual acuity (LogMAR) was worse in older graders, and the rate of eyeglass use was higher in older graders than in younger graders. The prevalence of DED was $4 \%$ in younger graders and $9.1 \%$ in older graders, which was a statistically significant difference $(P=0.03)$. The rate of smartphone use was $50.9 \%$ in younger graders compared to $65.1 \%$ in older graders. This was also statistically significant $(P<0.001)$ (Table 3$)$.

The mean daily duration of smartphone use was $0.57 \pm$ $0.76 \mathrm{~h}$ in younger graders and $1.00 \pm 1.06 \mathrm{~h}$ in older graders $(P<0.001)$, and the mean daily duration of computer use was $0.74 \pm 0.37 \mathrm{~h}$ in younger graders and $0.83 \pm$ $0.35 \mathrm{~h}$ in older graders $(P<0.01)$. These differences were all statistically significant. The daily duration of learning was $2.08 \pm 0.95 \mathrm{~h}$ in younger graders and $2.63 \pm 1.00 \mathrm{~h}$ in older graders $(P<0.001)$. The daily duration of outdoor activity was $2.49 \pm 1.07 \mathrm{~h}$ in younger graders and $1.94 \pm$ $1.07 \mathrm{~h}$ in older graders $(P<0.001)$. Region of residence, mean daily TV watching and sleep duration did not show significant differences between the two groups (Table 3).

\section{Analysis of risk factors and protective factors for pediatric DED}

We performed logistic regression analysis to investigate DED risk factors and protective factors. The mean daily duration of smartphone use was $3.18 \pm 0.97 \mathrm{~h}$ in the
Table 3 Comparative analysis of pediatric DED between younger (1st to 3rd) and older (4th to 6th) grade

\begin{tabular}{llll}
\hline & Younger grade & Older grade & $P$ \\
\hline Subjects (N) & 452 & 464 & \\
Region of Urban (\%) & 69.0 & 68.5 & $0.92^{\mathrm{a}}$ \\
Dry Eye Disease (\%) & 4 & 9.1 & $0.03^{\mathrm{a}}$ \\
Visual Acuity (LogMAR) & $0.28 \pm 0.42$ & $0.41 \pm 0.48$ & $<0.001^{\mathrm{b}}$ \\
SE (diopter) & $-1.77 \pm 2.12$ & $-2.39 \pm 2.11$ & $<0.001^{\mathrm{b}}$ \\
Use of Glass (\%) & 31 & 48.3 & $<0.001^{\mathrm{a}}$ \\
Related with DED Risk Factors & & & \\
$\quad$ Use of Smartphones (\%) & 50.9 & 65.1 & $<0.001^{\mathrm{a}}$ \\
$\begin{array}{l}\text { Smartphone use/day (hours) } \\
\text { Computer use/day (hours) }\end{array}$ & $0.57 \pm 0.74 \pm 0.37$ & $1.00 \pm 1.06$ & $<0.001^{\mathrm{b}}$ \\
TV use/day (hours) & $1.03 \pm 0.52$ & $1.13 \pm 0.55$ & $0.06^{\mathrm{b}}$ \\
$\begin{array}{l}\text { Learning/day (hours) } \\
\text { Related with DED Protective Factors }\end{array}$ & $2.08 \pm 0.95$ & $2.63 \pm 1.00$ & $<0.001^{\mathrm{b}}$ \\
Sleep/day (hours) & $7.93 \pm 0.83$ & $7.81 \pm 0.87$ & $0.11^{\mathrm{b}}$ \\
Outdoor activity/day (hours) & $2.49 \pm 1.07$ & $1.94 \pm 1.07$ & $<0.001^{\mathrm{b}}$ \\
\hline
\end{tabular}

$D E D$ dry eye disease, $S E$ spherical equivalent, logMAR logarithm of the minimum angle of resolution, $T V$ television

aby Pearson's Chi-square test, ${ }^{b}$ By independent $t$ test

DED group compared with $0.62 \pm 0.68 \mathrm{~h}$ in the normal group $(\mathrm{OR}=13.07, P<0.001)$ (Table 4$)$.

The mean daily duration of outdoor activity was $1.47 \pm$ $0.32 \mathrm{~h}$ in the DED group compared with $2.27 \pm 1.12 \mathrm{~h}$ in the normal group $(\mathrm{OR}=0.33, P<0.01)$. Mean daily duration of computer use, TV watching, learning and sleep did not relate to DED variably between the two groups (Table 4).

\section{Comparative analysis before and after cessation of smartphone use over 4 weeks in the DED group}

We performed comparative analysis before and after cessation of smartphone use over 4 weeks in the DED group. Punctate epithelial erosion improved after cessation of smartphone use from 93.3 to $0 \%(P<0.001)$. Tear break-up time also improved after cessation from $10.00 \pm 3.25 \mathrm{~s}$ to $11.33 \pm 2.29 \mathrm{~s}(P<0.001)$. OSDI score was decreased after smartphone cessation from 30.74 \pm 13.36 points to $14.53 \pm 2.23$ points $(P<0.001)$. Consequently, the rate of DED decreased from 100 to $0 \%$ after smartphone cessation over 4 weeks $(P<0.001)$ (Table 5$)$.

\section{Discussion}

In a previous study, the recent increase in smartphone use caused an increase in reports of symptoms such as irritation, burning sensation, conjunctival injection, decreased visual acuity, strain, and fatigue [11]. To the best of our knowledge, there have been no previous comparative studies of pediatric DED according to region and age. In the Republic of Korea, the internet is well-developed and 
Table 4 Analysis of risk factors and protective factors for pediatric DED

\begin{tabular}{|c|c|c|c|c|}
\hline & Normal & DED & OR $(95 \% \mathrm{Cl})$ & $P$ \\
\hline \multicolumn{5}{|l|}{ Related with DED Risk Factors } \\
\hline Smartphone use/day (hours) & $0.62 \pm 0.68$ & $3.18 \pm 0.97$ & 13.07 (5.99-28.52) & $<0.001^{a}$ \\
\hline Computer use/day (hours) & $0.76 \pm 0.34$ & $1.10 \pm 0.53$ & $0.94(0.20-4.42)$ & $0.94^{\mathrm{a}}$ \\
\hline TV use/day (hours) & $1.07 \pm 0.54$ & $1.18 \pm 0.40$ & $0.20(0.03-1.43)$ & $0.11^{\mathrm{a}}$ \\
\hline Learning/day (hours) & $2.31 \pm 1.02$ & $3.10 \pm 0.50$ & $2.51(0.90-7.03)$ & $0.08^{\mathrm{a}}$ \\
\hline \multicolumn{5}{|c|}{ Related with DED Protective Factors } \\
\hline Sleep/day (hours) & $7.88 \pm 0.86$ & $7.70 \pm 0.61$ & $0.63(0.22-1.77)$ & $0.38^{\mathrm{a}}$ \\
\hline Outdoor activity/day (hours) & $2.27 \pm 1.12$ & $1.47 \pm 0.32$ & $0.33(0.14-0.79)$ & $<0.01^{\mathrm{a}}$ \\
\hline
\end{tabular}

$D E D$ dry eye disease, $O R$ odds ratio, $\mathrm{Cl}$ confidence interval

${ }^{a}$ By Multivariate binominal logistic regression analysis

widely available, so it is common for children to use VDT to view media and internet content. Additionally, the rate of smartphone use has rapidly increased in Korean populations, especially among adolescents and school children.

A reduced blink rate during continuous smartphone use causes faster evaporation of the tear film, which may then lead to DED. In a previous study, we noted video display terminal use and reading reduced the blink rate to $5-6 / \mathrm{min}(1 / 3$ of the resting state rate) and promoted tear film evaporation and accommodation [12-15]. This may lead to ocular fatigue and myopic shift. Here, we pose the possibility that pediatric smartphone use and reading induces a lower blink rate, which may contribute to or cause pediatric DED. This comparative study of pediatric DED associated with smartphone use evaluates inter-region and inter-age variables. Logistic regression analysis was performed to investigate the risk and protective factors associated with pediatric DED.

The rate of smartphone use was significantly higher in the DED group than the control group. Continuous smartphone use is thought to decrease the blink rate and promote evaporation of the tear film, thus inducing DED. There was a significant difference in the mean

Table 5 Comparative analysis before and after cessation of smartphone use for 4 weeks in the DED group

\begin{tabular}{llll}
\hline & $\begin{array}{l}\text { Control } \\
(N=30)\end{array}$ & $\begin{array}{l}\text { Cessation of } \\
\text { smartphone }(N=30)\end{array}$ & $P$ \\
\hline PEE (\%) baseline & 100 & 93.3 & $0.50^{\mathrm{a}}$ \\
After 4 weeks & 86.7 & 0 & $<0.001^{\mathrm{a}}$ \\
TBUT (sec) baseline & $9.20 \pm 1.93$ & $10.00 \pm 3.25$ & $0.42^{\mathrm{c}}$ \\
After 4 weeks & $9.47 \pm 1.88$ & $11.33 \pm 2.29$ & $<0.001^{\mathrm{b}}$ \\
OSDI score baseline & $35.76 \pm 14.80$ & $30.74 \pm 13.36$ & $0.34^{\mathrm{c}}$ \\
After 4 weeks & $30.13 \pm 13.22$ & $14.53 \pm 2.23$ & $<0.001^{\mathrm{b}}$ \\
DED (\%) baseline & 100 & 100 & \\
4 weeks & 86.7 & 0 & $<0.001^{\mathrm{a}}$
\end{tabular}

PEE punctate epithelial erosion, TBUT tear break up time, OSDI ocular surface disease index, $D E D$ dry eye disease

a'By Pearson's Chi-square test, 'by Repeated measure ANOVA,

'By Independent t-test duration of outdoor activity and learning between the two groups; however, on logistic regression analysis, the mean duration of smartphone use $(\mathrm{OR}=13.07)$ was the only strong risk factor for pediatric DED. The mean duration of computer use, TV watching and learning was not significantly different on multivariate logistic regression analysis. In addition, increased outdoor activity time reduced the rate of pediatric $\mathrm{DED}(\mathrm{OR}=0.33)$.

A greater proportion of students in the DED group were urban versus rural residents. We suggest that that the difference in smartphone use between regions led to the difference in DED prevalence. The rate of eyeglass use was higher in urban students and visual acuity (LogMAR) was better in the rural group. The rate of DED prevalence was higher in the urban group compared to the rural group, and the mean daily duration of smartphone use, computer use, and learning was significantly longer in urban populations. Outdoor activity time was longer in the rural group. The longer duration of smartphone use, computer use and learning relative to low outdoor activity may have increased the prevalence of DED and the rate of eyeglass use in urban populations.

DED prevalence was also found to be higher in females than males. We surveyed our study population to identify these differences in Korean children. In our study, DED prevalence in children was higher in females; however, there was no statistical significance. There was no difference between the two groups with regard to VDT use. In general, DED prevalence was higher in female adults compared to male adults.

The mean age was higher in the DED group than in the normal group, and DED prevalence was higher in older graders than younger graders. The rate of smartphone use was higher and the mean daily duration of VDT was longer in older grades, which may have led to the higher DED prevalence in older graders.

Smartphones are used with short watching distances due to their small LED screens, thus inducing ocular fatigue, glare, and irritation. VDT is associated with an 
increased incidence of DED and decreased visual acuity. Gur [16] et al. reported that VDTs require reduced accommodation and fusional convergence and induce a -0.12 diopter myopic shift.

A limitation of our study is that only one school in each region was surveyed. If we surveyed a greater number of schools in urban or rural areas, our data would be more representative. Our study was aimed at school children, so there may have been difference in self-reporting and expression of ocular discomfort and comprehension of the questionnaire between younger graders and older graders, which could have affected our estimates of DED prevalence. We did not consider a myriad of other factors, including environmental and socioeconomic status. We have shown an association and that in those who have dry eyes we have shown that cessation of smartphone use improves signs and symptoms; however, there are many other variables that we have not assessed. In a smartphone cessation study, it would be better to use controls who had subjective complaints or higher OSDI scores and no obvious disease on clinical exams to rule out accommodative fatigue or placebo effect.

We used subjective symptom criteria (modified OSDI score) and objective signs (low TBUT or PEE) to diagnose DED. These criteria were based on adults and subjective signs of DED were not obvious. Thus, the true prevalence of DED in school children may be increased or decreased due to the use of adult-targeted diagnostic criteria. In our previous study, we confirmed that DED coexists with allergic conjunctivitis [17]; however, because DED and ocular allergies have similar symptoms, it was difficult to separate children with DED from those with ocular allergies $[18,19]$. Thus, the prevalence of DED may have been affected by the prevalence of allergies in our study population. We did not consider confounding factors such as socioeconomic status, family composition, dual income family status, or concurrent artificial tear use.

\section{Conclusion}

In conclusion, increased VDT use such as smartphones or computers in Korean children was found to be associated with the occurrence of ocular surface symptoms. Increased use of smartphones is a serious issue that may result in ocular problems: the prevalence of DED was $6.6 \%$. The mean duration of VDT use per day, especially smartphone use, was greater in the DED group than in the normal group. Longer daily smartphone use may be a risk factor for DED. The prevalence of DED was higher in the urban group and in older children. Older-grade children in the urban group used smartphones for longer time periods than younger-grade children in rural areas. DED prevalence was higher in older grade children from urban groups compared to younger grade children in rural groups. After cessation of smartphone use for
4 weeks in DED patients, both subjective symptoms and objective signs improved.

Therefore, close observation and caution during VDT use, especially smartphones, is recommended for older children in urban areas. DED in children must be detected early and should be treated with appropriate medical and environmental interventions and education.

\section{Additional file}

Additional file 1: OSDI, questionnaire and protocol. This file included OSDI index, ophthalmologic questionnaire and study protocol. (DOCX 83 kb)

\section{Abbreviations}

$\mathrm{Cl}$ : Confidence interval; DED: Dry eye disease; logMAR: logarithm of the minimum angle of resolution; OR: Odds ratio; OSDI: Ocular surface disease index; SE: Spherical equivalent; TBUT: Tear break up time; TV: Television; VDT: Video display terminal

\section{Acknowledgements}

We thank the teachers of the two elementary schools and students that contributed to the database used in this study. We also acknowledge the clinical assistance provided by Lee DI, Heo J, Choi SU, Lee MY.

Funding

None.

Availability of data and materials

All data are available upon request.

Authors' contributions

JHM: 1st author, study design, data collection, analysis and interpretation of data, draft writing. NJM: study design, data collection, analysis and interpretation of data, draft writing, review, manuscript approval. KWK: analysis and interpretation of data, draft writing, review. All authors read and approved the final manuscript.

\section{Competing interests}

The authors declare that they have no competing interests.

\section{Consent for publication}

We also confirmed that parents had seen the manuscript and patient data and agreed to its publication in a journal.

\section{Ethics approval and consent to participate}

The study protocol was in accordance with the Declaration of Helsinki and was approved by the institutional review board of Chung-Ang University Hospital, Seoul, Korea.

Both parents and children themselves provided written informed consent after being given a detailed explanation of the study. We confirmed that parents had seen the manuscript and patient data and agreed to its publication in a journal.

Received: 23 March 2016 Accepted: 14 October 2016 Published online: 28 October 2016

\section{References}

1. McCarty CA, Bansal AK, Livingston PM, Stanislavsky YL, Taylor HR. The epidemiology of dry eye in Melbourne, Australia. Ophthalmology. 1998;105: 1114-9.

2. Ang CK, Mohidin N, Chung KM. Effects of wink glass on blink rate, nibut and ocular surface symptoms during visual display unit use. Curr Eye Res. 2014;39:879-84.

3. Yaginuma $Y$, Yamada $H$, Nagai $H$. Study of the relationship between lacrimation and blink in VDT work. Ergonomics. 1990;33:799-809. 
4. Yang WJ, Yang YN, Cao J, Man ZH, Yuan J, Xiao X, Xing YQ. Risk factors for Dry Eye syndrome: a retrospective case-control study. Optom Vis Sci. 2015;92:199-205.

5. González-Pérez M, Susi R, Antona B, Barrio A, González E. The ComputerVision Symptom Scale (CVSS17): development and initial validation. Invest Ophthalmol Vis Sci. 2014;55:4504-11.

6. Alves M, Dias AC, Rocha EM. Dry eye in childhood: epidemiological and clinical aspects. Ocul Surf. 2008;6:44-51.

7. Moon JH, Lee MY, Moon NJ. Association between video display terminal use and dry eye disease in school children. J Pediatr Ophthalmol Strabismus. 2014;51:87-92.

8. Krachmer JH, Mannis MJ, Holland EJ. Cornea. 3rd ed. Philadelphia: Elsevier; 2011.

9. Bron AJ, Evans VE, Smith JA. Grading of corneal and conjunctival staining in the context of other dry eye tests. Cornea. 2003;22:640-50.

10. The definition and classification of dry eye disease: report of the Definition and Classification Subcommittee of the International Dry Eye WorkShop (2007). Ocul Surf. 2007; 5: 75-92.

11. Bergqvist UO, Knave BG. Eye discomfort and work with visual display terminals. Scand J Work Environ Health. 1994;20:27-33.

12. Freudenthaler $\mathrm{N}$, Neuf $\mathrm{H}$, Kadner $\mathrm{G}$, Schlote $\mathrm{T}$. Characteristics of spontaneous eyeblink activity during video display terminal use in healthy volunteers. Graefes Arch Clin Exp Ophthalmol. 2003:241:914-20.

13. Nakamori K, Odawara M, Nakajima T, Mizutani T, Tsubota K. Blinking is controlled primarily by ocular surface conditions. Am J Ophthalmol. 1997; 124:24-30.

14. Korb DR, Baron DF, Herman JP, Finnemore VM, Exford JM, Hermosa JL, Leahy CD, Glonek T, Greiner JV. Tear film lipid layer thickness as a function of blinking. Cornea. 1994;13:354-9.

15. Fenga C, Aragona P, Di Nola C, Spinella R. Comparison of ocular surface disease index and tear osmolarity as markers of ocular surface dysfunction in video terminal display workers. Am J Ophthalmol. 2014;158:41-8.

16. Gur S, Ron S, Heicklen-Klein A. Objective evaluation of visual fatigue in VDU workers. Occup Med (Lond). 1994:44:201-4.

17. Kim TH, Moon NJ. Clinical correlations of dry eye syndrome and allergic conjunctivitis in Korean children. J Pediatr Ophthalmol Strabismus. 2013;50:124-7.

18. Fujishima H, Toda I, Shimazaki J, Tsubota K. Allergic conjunctivitis and dry eye. Br J Ophthalmol. 1996;80:994-7.

19. Toda I, Shimazaki J, Tsubota K. Dry eye with only decreased tear break-up time is sometimes associated with allergic conjunctivitis. Ophthalmology. 1995;102:302-9.

\section{Submit your next manuscript to BioMed Central and we will help you at every step:}

- We accept pre-submission inquiries

- Our selector tool helps you to find the most relevant journal

- We provide round the clock customer support

- Convenient online submission

- Thorough peer review

- Inclusion in PubMed and all major indexing services

- Maximum visibility for your research

Submit your manuscript at www.biomedcentral.com/submit 